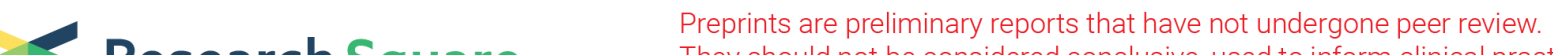 $\begin{array}{ll}\text { Research Square } & \text { They should not be considered conclusive, used to inform clinical practice, } \\ \text { or referenced by the media as validated information. }\end{array}$
}

\section{Anterior Reduction and C1-ring Osteosynthesis With Jefferson-fracture Reduction Plate (JeRP) via Transoral Approach for Unstable Atlas Fractures}

\section{Qiang Tu}

PLA General Hospital of Southern Theatre Command: People's Liberation Army General Hospital of Southern Theatre Command

\section{Hu Chen}

PLA General Hospital of Southern Theatre Command: People's Liberation Army General Hospital of Southern Theatre Command

Zhan Li

Guangzhou University of Chinese Medicine

\section{Yuyue Chen}

PLA General Hospital of Southern Theatre Command: People's Liberation Army General Hospital of Southern Theatre Command

\section{Aihong Xu}

PLA General Hospital of Southern Theatre Command: People's Liberation Army General Hospital of Southern Theatre Command

\section{Changrong Zhu}

PLA General Hospital of Southern Theatre Command: People's Liberation Army General Hospital of

Southern Theatre Command

\section{Xianhua Huang}

PLA General Hospital of Southern Theatre Command: People's Liberation Army General Hospital of

Southern Theatre Command

\section{Xiangyang Ma}

PLA General Hospital of Southern Theatre Command: People's Liberation Army General Hospital of

Southern Theatre Command

\section{Jianhua Wang}

PLA General Hospital of Southern Theatre Command: People's Liberation Army General Hospital of Southern Theatre Command

\section{Kai Zhang}

PLA General Hospital of Southern Theatre Command: People's Liberation Army General Hospital of Southern Theatre Command Jianzhong Xu

Southwest Hospital, Third Military Medical University 
Hong Xia ( $\sim$ gzxiahong2@126.com )

Department of Orthopaedic Surgery, PLA General Hospital of Southern Theatre Command: People's Liberation Army General Hospital of Southern Theatre Command, 510010, Guangdong, China https://orcid.org/0000-0003-3651-8410

\section{Research article}

Keywords: atlas, fractures, transoral approach;surgery, osteosynthesis

Posted Date: January 29th, 2021

DOI: https://doi.org/10.21203/rs.3.rs-155382/v1

License: (c) (i) This work is licensed under a Creative Commons Attribution 4.0 International License.

Read Full License 


\section{Abstract}

Objective:To introduce a novel transoral instrumentation in the treatment of unstable fractures of atlas.

Methods: From January 2008 to May 2018, 22 patients with unstable C1 fractures were retrospective analyzed, who received Jefferson-fracture reduction plate (JeRP) via transoral approach. The case history and the radiographs before and after surgery were noted. The type of fracture, the reduction of the fracture and position of the internal fixation were assessed by preoperative and postoperative CT scans.

RESULTS: All 22 patients successfully underwent anterior C1-ring osteosynthesis using JeRP system, with a follow-up of $26.84 \pm 9.23$ months. Among them, 9 patients had transverse atlantal ligament (TAL) injury, including 3 in Dickman type I and 6 in type II. The preoperative lateral mass displacement (LMD) decreased from $7.13 \pm 7.08 \mathrm{~mm}$ to $1.02 \pm 0.65 \mathrm{~mm}$ after operation. Bone union was achieved in all patients without implant failure or loss of reduction. There were no surgery-related complications, such as wound infection, neurological deficit, or vertebral artery injury. However, 3 patients with Dickman type I TAL injury occured atlantoaxial dislocation 3 months postoperatively without any neurological symptoms or neck pain.

CONCLUSIONS: Transoral C1-ring osteosynthesis with JeRP is an effective surgical strategy of unstable atlas fractures, achieving direct and satisfactory reduction safely. The primary indication for JeRP system is an unstable fracture (Gehweiler type I/III) or/ and TAL injury (Dickman type II).

\section{Background}

The atlas has a unique ring structure with no vertebral body or spinous processes, which provides the flexibility and maximum range of motion (ROM) compared to any other vertebra of the spine ${ }^{[1]}$. As a transitional structure, it is an indispensable part of craniovertebral junction,which allows axial loading transferred from the occiput to axis. The junctions of the lateral mass connected to both the anterior and posterior arches are relatively thin, which are the weakest points of $\mathrm{C} 1$ and the most likely sites of fracture ${ }^{[2]}$. Thus, the fracture with two or more breaks commonly occurred in the C1-ring ${ }^{[3]}$. In general, the type of atlas fracture can be divided into stables and unstable according to the integrity of transverse atlantal ligament (TAL) and adjacent vertebrae.Unstable $\mathrm{C} 1$ fractures are characterized by high-grade transverse spread of lateral mass on open-mouth radiograph.

Until now, posterior atlantoaxial and occipitocervical fusion techniques have been widely used in the treatment of it and have achieved satisfactory results ${ }^{[4]}$. However, posterior fusion techniques decrease the ROM of the craniocervical junction. As to this problem, anterior C1-ring osteosynthesis is an appropriate option to perform ORIF via transoral approach with safety and effectiveness ${ }^{[5]}$. However,surgical site infection is a worrying complication,making surgeons preferred the posterior C1-ring osteosynthesis. Nevertheless, posterior C1-ring osteosynthesis can't completely reduce and stabilize C1 anterior arch fractures.In 2004,Ruf ${ }^{[6]}$ first reported a C1-C2 rotation function-preserving technique of C1 
osteosynthesis via transoral approach, which not only enables an anatomic reconstruction of anterior

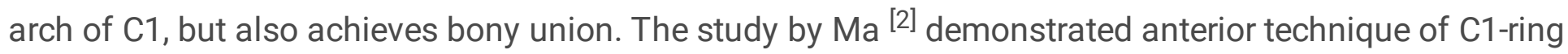
ORIF with direct manipulation at the lateral masses was safe and effective for treatment of highly unstable cases. However, the internal fixation device used in C1-ring osteosynthesis is not a special instrumentation for atlas fractures, and it is unlikely to achieve near anatomical reduction for $\mathrm{C} 1 \mathrm{fracture}$ in direct and safety especially for the $\mathrm{C} 1$ anterior arch fractures. To our knowledge,there is no report on special apparatus for $\mathrm{C} 1$ osteosynthesis via transoral approach.

In this study, we described a novel Jefferson-fracture reduction plate (JeRP) system (Wego Corporation, China) via transoral approach. It is originally designed and clinically applied to treat unstable atlas fracture, for preserving motion-function of atlantoaxial joint.

\section{Methods}

This research program was approved by the institutional review committee of PLA General Hospital of Southern Theatre Command: People's Liberation Army General Hospital of Southern Theatre Command. Each patient signed a medical informed consent documents preoperatively.

From Jan 2008 to May 2018, 22 patients with unstable C1 fractures were retrospective analyzed, who received JeRP via transoral approach. Patients with fractures of the adjacent vertebrae of the axis or occipital condyle were excluded. All patients suffered neck pain, stiffness and limited motion of neck movement. None of the them had neurological deficit.On admission,all the patients underwent x-ray film,CT scans and MRI preoperatively (Fig. 1,2). The lateral mass displacement(LMD) of atlas was measured from the coronal reconstructed view of the CT scans. Integrity of the TAL was evaluated by preoperative MRI. All patients underwent $4-6 \mathrm{~kg}$ skull traction preoperatively and intraoperatively in the hope that the displaced lateral mass might close at least partially.

\section{Design of the JeRP system}

The JeRP-system consists of titanium alloy plate,self-tapping screws,specialized repositor and corresponding surgical instruments (Fig. 3). The JeRP-plate shapes like an "L",and is consistent with the anatomical morphology in front of the atlas, and the lateral mass end of it is provided with two circular fixing holes fixed to the lateral mass of $\mathrm{C} 1$. On the side views,the plate has a slight arc,fitting the physiological curvature of C1.Additionally, there are two types of L-shaped plates, left and right, which are used for $\mathrm{C} 1$ fractures with the fracture line closed to the left or right lateral mass,respectively. The middle of it is a long-elliptic hole in which a provisional reduction screw can slide. Specialized repositor is worth noting. With the help of it,compression force is gently applied to pull together the fractured ends for reduction. The working principle of JeRP-system in the treatment of $\mathrm{C} 1$ fracture is shown in Fig. 4.

\section{Surgical Technique}


All surgeries were performed by senior spine surgeons. Under general anesthesia with transnasal endotracheal intubation,the patient was placed in a supine position with skull traction with a weight of 4$6 \mathrm{~kg}$. Codman oral retractor used to expose the oropharynx,a 3-4 cm longitudinal midline incision along the posterior pharyngeal wall was made,and soft tissue was dissected to fully exposed the surgical field, including anterior arch, fragment bone and lateral mass of atlas (Fig. 5A). According to the preoperative CT measurement,the ideal entry point, the trajectory of the screw and the length of the JeRP-plate were determined. The anterior tubercle of $\mathrm{C} 1$ was polished with high-speed drill. After removing soft tissue between bony fragments, the JeRP -plate was placed in anterior aspect of atlas (Fig. 5B). By palpating the medial edge and lower margin of the lateral mass,we could determine the optimal entry point of the screws. First, one lateral mass screw close to the fracture line was installed and tightened for stabilized the plate. Then, a provisional reduction screw was placed in the anterior arch of $\mathrm{C} 1$, which could be moved in the runner of the plate,and the nut was about $3 \mathrm{~mm}$ away from the bone surface of the anterior arch.

The specialized repositor was installed between a provisional screw and the complex comprised with the lateral mass and the plate (Fig. 5C, D). Compression force was gently applied via the special repositor to pull together the fractured ends and to make it fit tightly. Next, the other lateral mass screws were tightened subsequently, the temporary screw removing, and the entire reduction fixation process was complete. Finally, the JeRP-plate was affixed to the anterior arch,and satisfactory reduction of fracture and position of internal fixation were confirmed by intraoperative fluoroscopy.After JeRP-implant procedure, the wound was soaked with povidone-iodine for 15 minutes and was closed in muscular and mucosal layers respectively with interrupted sutures.

\section{Postoperative treatment}

Transnasal endotracheal catheter was maintained for 2 days after the operation, and a nasogastric feeding tube was kept for 1 week. After the removal of the nasogastric tube, fluid diet was started and normal diet was started 3 weeks postoperatively. Antibiotics were given intravenously as preventive interventions in all patients for 5-7 days after surgery and the neck collar was kept for 3 months. Postoperative CT were used to assess the efficiency of the $\mathrm{C} 1$ reduction and the accuracy of the screw placement. What's more, CT scans and X-ray film of cervical spine were performed at three, six and twelve months after surgery to evaluate bony healing, reduction and stability of the $\mathrm{C} 1-\mathrm{C} 2$ (Fig. 6). At the final follow-up, the atlanto-dens interval (ADI) was calculated based on flexion-extension radiographs. Also, visual analogue scale (VAS) was performed to evaluate the degree of neck pain.

\section{Results}

The demographic data of patients were listed in Table 1. In all patients, the incision of the posterior pharyngeal wall healed well, and no infection or dehiscence occurred. No vertebral artery or spinal cord injury occurred during the operation. According to Gehweiler's classification system, 7 of 22 patients presented with type $\otimes$ fractures and 15 of 22 patients had type $\otimes$ fractures. C1-rings was violated into few parts, including 2 separate fragments (7 patients), 3 fragments ( 13 patients), and 4 fragments ( 2 patients). 9 patients had certain TAL injury (3 of type I and the other of type II based on Dickman's 
classification), while other 13 patients had TAL injury or not were uncertain. The surgical and clinical outcomes can be listed in Tables 2 and 3.All operations were completed successfully completed without surgery-related complications, such as wound infection, neurological deficit,or vertebral artery injury.The preoperative LMD decreased from $7.13 \pm 7.08 \mathrm{~mm}$ to $1.02 \pm 0.65 \mathrm{~mm}$ after operation. Bone union was achieved in all patients without implant failure or loss of reduction. At the final follow-up, the mean VAS scores was $0.28 \pm 0.13$ points. What's more, all patients' upper cervical spine rotation range was well preserved. However, 3 patients had an ADI of more than $4 \mathrm{~mm}$ at the last follow-up without any neurological symptoms or neck pain. 1 patient had limited cervical movement due to the penetration of the atlanto-occipital facet with lateral mass screws, but no obvious pain and no special treatment.

Table 1

Demographic Data

\begin{tabular}{|c|c|}
\hline Variables & $\mathbf{N}$ \\
\hline Gender (Male,n) & $22(12)$ \\
\hline Age (years) & $48.14 \pm 9.66$ \\
\hline Follow-up time (months) & $26.84 \pm 10.23$ \\
\hline \multicolumn{2}{|l|}{ Type of fractures (n) } \\
\hline Type $\otimes$ & 7 \\
\hline Type $\otimes$ & 15 \\
\hline Other types & 0 \\
\hline \multicolumn{2}{|l|}{ TAL injury } \\
\hline Type $\otimes$ & 3 \\
\hline Type II & 6 \\
\hline Uncertain & 13 \\
\hline
\end{tabular}


Table 2

Surgical outcomes

\section{Variables}

Surgical $\quad 181.32 \pm 185.00$

time(min)

Blood

$144.21 \pm 155.67$

loss $(\mathrm{ml})$

Hospital $\quad 19.00 \pm 10.55$

stay (d)

Complications 1 patient had limited cervical movement due to the penetration of the atlantooccipital facet with lateral mass screws, but no obvious pain and no special treatment.No surgery-related complications,such as wound infection, neurological deficit, or vertebral artery injury.

Table 3

Clinical outcomes

\begin{tabular}{|lllll|}
\hline Parameters & Preoperative & Postoperative & Follow-up & P \\
\hline LMD $(\mathrm{mm})$ & $7.13 \pm 7.08$ & $1.02 \pm 0.65$ & $0.53 \pm 0.21$ & $\varangle 0.0001$ \\
\hline VAS & $7.42 \pm 3.92$ & $2.17 \pm 1.33$ & $0.28 \pm 0.13$ & $\varangle 0.0001$ \\
\hline Complications & $\begin{array}{l}\text { 3 patients with Dickman type I TAL injury occured atlantoaxial dislocation 3 months } \\
\text { postoperatively without any neurological symptoms or neck pain }\end{array}$ & \\
\hline
\end{tabular}

\section{Discussion}

Most of stable atlas fractures can be treated conservatively ${ }^{[7]}$. However, as to unstable $\mathrm{C} 1$ fracture, surgical strategies have become the standard of care. Unstable atlas fracture usually accompanies TAL injury, behaving as separation of the lateral masses, and subluxation or dislocation of atlantoaxial joint ${ }^{[5]}$.There is also a point that atlas fractures, not $\mathrm{C} 1$ posterior arch fractures, destroyed the stability of the upper cervical spine. The spinal cord is under danger since the displacement of bone fragments or atlantoaxial dislocation, which resulting in severe complications like paraplegia even death $[8,9]$. Treatment aspires to reduce the fracture, correct dislocated fracture, stabilize the atlantoaxial joint, and preserve the maximum ROM of the upper cervical spine ${ }^{[10,11]}$. However, the surgical strategies of unstable $\mathrm{C} 1$ fractures is still controversial.

\section{Value of C1-ring osteosynthesis in unstable atlas fracture}

Conservative therapy in unstable atlas fracture for several months may lead to severe discomfort and high incidence of bony nonunion ${ }^{[12]}$.Simultaneously,the inconsistency and mechanical instability of occipitocervical junction may restricted motion and cause continuous neck pain ${ }^{[13]}$. To date,the posterior atlantoaxial or occipitocervical fusion is supposed to be the main surgical method,but the motion of 
upper cervical spine was sacrificed. The ideal treatment method is limited fixation without restricting the ROM of upper cervical spine ${ }^{[14]}$. For this, many spine surgeons gave up the posterior fusion and recommend $\mathrm{C} 1$-ring osteosynthesis ${ }^{[15,16]}$. The question regarding of relationship between $\mathrm{C} 1$-ring osteosynthesis and integrity of the TAL is still controversial.Traditionally, the integrity of TAL is key in determining the stability of $\mathrm{C} 1$ fractures. Rule of Spence ${ }^{[17]}$ showed that total LMD over $6.9 \mathrm{~mm}$ on openmouth radiographs correlated with rupture to TAL has important clinical value in determining whether surgical intervention is needed and is currently being questioned. What's more,there are other tissues that help maintain C1-C2 stability and restrict motion notwithstanding rupture of TAL.Some scholars' founding shown that the significance of axial ligamentous tension of craniocervical junction has been underrated ${ }^{[18]}$. Because of its unique anatomical structure and biomechanical environment, occipitocervical junction mainly stabilized by the ligamentous complex of $\mathrm{C} 0-\mathrm{C} 1-\mathrm{C} 2^{[13]}$. Previous literature had showed that $\mathrm{C} 1$ burst fractures are axial load entities and better to maintain the integrity of secondary stabilizers comprised the alar ligaments, facet capsule and neck musculature. C1-ring osteosynthesis techniques is able to restore the axial tension of ligamentous complex of $\mathrm{CO}-\mathrm{C} 1-\mathrm{C} 2$ through reduction of fracture. Studies have shown that even with the rupture of TAL,C1-ring osteosynthesis can provide sufficient stability under physiological load. Thus, incompetence of TAL may not be a contraindication to $\mathrm{C} 1$-ring osteosynthesis. The conventional definition of $\mathrm{C} 1$ instability based on the integrity of TAL underestimates the number of fractures requiring surgical intervention and overestimates the number requiring $\mathrm{C} 1-\mathrm{C} 2$ fusion.

\section{Advantage of anterior C1-ring osteosynthesis using JeRP system}

C1-ring osteosynthesis using both posterior approach and transoral approach has been published ${ }^{[18]}$. With posterior $\mathrm{C} 1$-ring osteosynthesis techniques,posterior arch fracture and the lateral mass displacement could be satisfactorily reduced by the compression force on the end of bilateral mass screws. However,it makes the front of the lateral mass screw swing laterally, leading to insufficient reduction of anterior arch fracture of $\mathrm{C} 1^{[19]}$.

Anterior direct reduction of the atlas fractures promotes the rate of bony union of fractures by improving the integrity of $\mathrm{C}-\mathrm{C} 1-\mathrm{C} 2$ complex structure. This approach has a good safety profile,avoiding fusion of important motion segments, and restoring the $\mathrm{C} 0$-C2 height. Over last decade, results of transoral C1-ring osteosynthesis for unstable atlas fracture have been verified. However, many surgeons are hesitant about this technique,because of unfamiliarity with the transoral approach,the theoretical increased risk of infection. The universal shortcoming of transoral $\mathrm{C} 1$ osteosynthesis published previously is that reduction of $\mathrm{C} 1$ fracture is only an acceptable repair rather than anatomical reconstruction. The transoral C1-ring osteosynthesis is technically challenging, and there is no specific instrumentation devices or spinal implants designed for treating unstable atlas fracture. The main problem with current techniques is that the posterior pharyngeal soft tissue which is not thick enough to cover the plate or rod,thus increasing the risk of wound complications.Additionally,it is difficult to implement satisfactory reduction of atlas 
fracture in the deep and narrow space. Simultaneously,the end of the lateral mass screw via transoral approach is too high,which may easily cause the wound of the posterior pharyngeal wall to crack or postoperative dysphagia.In this study,the use of JeRP-system is introduced for anterior C1-ring osteosynthesis. The advantage of it lies in the use of a dedicated reduction instrument, which not only satisfies the reduction of the fracture end,but also can place the fixation screws in the fracture reduction state and the reduction instrument will not affect the screw placement. Generally,it is acceptable to follow the principle that the screws don't penetrate the edge of the lateral mass into the atlanto-occipital joint, and don't enter atlantoaxial joint.The lateral mass is wedge-shaped, with higher outside and lower inside. The insertion point of the screw is deviation inward, which easily leads to the screw entering joint. In our paper, 1 lateral mass screws were observed to enter atlanto-occipital joint.In the series, 22 patients had bone fusion, and wound infection and dehiscence had not been observed. The main advantage of JeRP-system is not only ideally reduction of $\mathrm{C} 1$ fracture performed via anterior approach, but also inserted plate and screws could not interfere with midline wound closure.The incongruency of the CO-C1 and $\mathrm{C} 1-\mathrm{C} 2$ joints is rectified, and the ligamentous tension band of craniocervical junction is regained as well.As far as we know,this new technology can minimize lateral mass displacement.JeRP-system appears to be an effective and safe method to deal with unstable $\mathrm{C} 1$ fractures, which achieve ideal bone fusion and motion-preserving of craniocerivcal junction.

The original intention of the JeRP-system designed is to be used for unstable $\mathrm{C} 1$ fractures with TAL intact,and its indication is very narrow. In the actual process, we also applied the JeRP-system to C1 fractures with TAL rupture,and achieved satisfactory results. Among them,3 patients with Dickman type I TAL injury occurred atlantoaxial dislocation postoperatively,while the patients with Dickman type II TAL injury had good effect. Hence, the primary indication for JeRP system is an unstable $\mathrm{C} 1$ fracture (Gehweiler type I/III) with or without TAL injury (Dickman type II).

\section{Conclusions}

Transoral C1-ring osteosynthesis with JeRP-system is a valid option of unstable $\mathrm{C} 1$ fractures, achieving direct and satisfactory reduction safely. Even if the TAL is ruptured,the atlantoaxial joint can remain relatively stable.TAL injury may not necessarily be an absolute contraindication for ORIF for altas fracture. However, further studies should be made to investigate the long-term effect of atlantoaxial instability.

Limitations of this paper are the lack quantified range of motion-preserving of atlantooccipital and atlantoaxial joints,small sample size,retrospective design and possibility of selection bias.

\section{Declarations}

Funding $\triangle$ Military Medical Science and Technology Youth Cultivation Program Incubation Project (NO.19QNP040); ; 奴e Science and Technology Planning Project of Guangzhou City 
(N0.201904010349) खPhD projects of Third Military Medical University(NO.JSKT201904). Army Logistics Research Project of PLA (CLB20J033). All the funders are QT.

\section{Conflicts of interest/Competing interests NO}

Availability of data and material Informed consent was obtained for publication of patient data. We have full control of all primary data and agree to allow the journal to review their data if requested.

\section{Code availability No}

Authors' contributions All authors contributed to the surgery that the data collected from. The first draft of the manuscript was written by QT and all authors commented on previous versions of the manuscript. Data collection and analysis were performed by HC. All authors read and approved the final manuscript.

\section{Consent for publication Not Applicable.}

\section{References}

1. Wang Jian, Zhou Yue, Zhang Zheng Feng et al. (2012) Direct repair of displaced anterior arch fracture of the atlas under microendoscopy: experience with seven patients. [J]. Eur Spine J, 21: 34751.

2. Ma Weihu, Xu Nanjian, Hu Yong et al.(2013) Unstable atlas fracture treatment by anterior plate C1ring osteosynthesis using a transoral approach. [J]. Eur Spine J, 22: 2232-9.

3. Zou Xiaobao, Ouyang Beiping, Wang Binbin et al.(2020) Motion-preserving treatment of unstable atlas fracture: transoral anterior C1-ring osteosynthesis using a laminoplasty plate. [J]. BMC Musculoskelet Disord, 21: 538.

4. Laubach Markus, Pishnamaz Miguel, Scholz Matti et al.(2020) Interobserver reliability of the Gehweiler classification and treatment strategies of isolated atlas fractures: an internet-based multicenter survey among spine surgeons. [J]. Eur J Trauma Emerg Surg, undefined: undefined.

5. Guo Wei, Lin Yang, Huang Jingwen et al.(2020) Treatment strategy of unstable atlas fracture: A retrospective study of 21 patients. [J]. Medicine (Baltimore), 99: e20153.

6. Ruf Michael, Melcher Robert, Harms Jürgen,(2004) Transoral reduction and osteosynthesis $\mathrm{C} 1$ as a function-preserving option in the treatment of unstable Jefferson fractures. [J]. Spine,29: 823-7.

7. Bednar Drew A, Almansoori Khaled A, (2016) Solitary C1 Posterior Fixation for Unstable Isolated Atlas Fractures: Case Report and Systematic Review of the Literature. [J] Global Spine J, 6: 375-82.

8. Fiedler Nora, Spiegl Ulrich J A, Jarvers Jan-Sven et al.(2020) Epidemiology and management of atlas fractures. [J]. Eur Spine J, 29: 2477-2483.

9. Schleicher Philipp, Scholz Matti, Kandziora Frank et al.(2019) Recommendations for the Diagnostic Testing and Therapy of Atlas Fractures. [J]. Z Orthop Unfall, 157: 566-573. 
10. Kandziora Frank, Scholz Matti, Pingel Andreas et al.(2018) Treatment of Atlas Fractures: Recommendations of the Spine Section of the German Society for Orthopaedics and Trauma (DGOU). [J] Global Spine J, 8: 5S-11S.

11. Smith Richard M, Bhandutia Amit K, Jauregui Julio J et al.(2018) Atlas Fractures: Diagnosis, Current Treatment Recommendations, and Implications for Elderly Patients. [J]. Clin Spine Surg, 31: 278-284.

12. Zhao Zhong-Sheng, Wu Guang-Wen, Lin Jie et al.(2019) Management of Combined Atlas Fracture with Type II Odontoid Fracture: A Review of 21 Cases. [J] Indian Journal of Orthopaedics., 53: 518524.

13. Zhang Yin-Shun, Zhang Jian-Xiang, Yang Qing-Guo et al.(2018) Posterior osteosynthesis with monoaxial lateral mass screw-rod system for unstable C1 burst fractures. [J]. Spine J, 18: 107-114.

14. Hu Yong, Xu Rong-Ming, Albert Todd $\mathrm{J}$ et al.(2014) Function-preserving reduction and fixation of unstable Jefferson fractures using a C1 posterior limited construct. [J]. J Spinal Disord Tech, 27: E219-25.

15. Ottenbacher Andy, Bettag Martin,(2017) Resolution of traumatic vertebral artery dissection and occlusion after repositioning and posterior C1-ring osteosynthesis of a displaced Jefferson burst fracture. [J]. Acta Neurochir (Wien), 159: 1561-1564.

16. Koller Heiko,Resch Herbert,Tauber Mark et al.(2010) A biomechanical rationale for C1-ring osteosynthesis as treatment for displaced Jefferson burst fractures with incompetency of the transverse atlantal ligament. [J]. Eur Spine J, 19: 1288-98.

17. Woods Rafeek O, Inceoglu Serkan, Akpolat Yusuf T et al.(2018) C1 Lateral Mass Displacement and Transverse Atlantal Ligament Failure in Jefferson's Fracture: A Biomechanical Study of the "Rule of Spence". [J]. Neurosurgery, 82: 226-231.

18. Shatsky Joshua, Bellabarba Carlo, Nguyen Quynh et al.(2016) A retrospective review of fixation of C1 ring fractures--does the transverse atlantal ligament (TAL) really matter? [J] .Spine J, 16: 372-9.

19. Kandziora Frank, Chapman Jens R, Vaccaro Alexander R et al.(2017) Atlas Fractures and Atlas Osteosynthesis: A Comprehensive Narrative Review. [J]. J Orthop Trauma, S81-

\section{Figures}



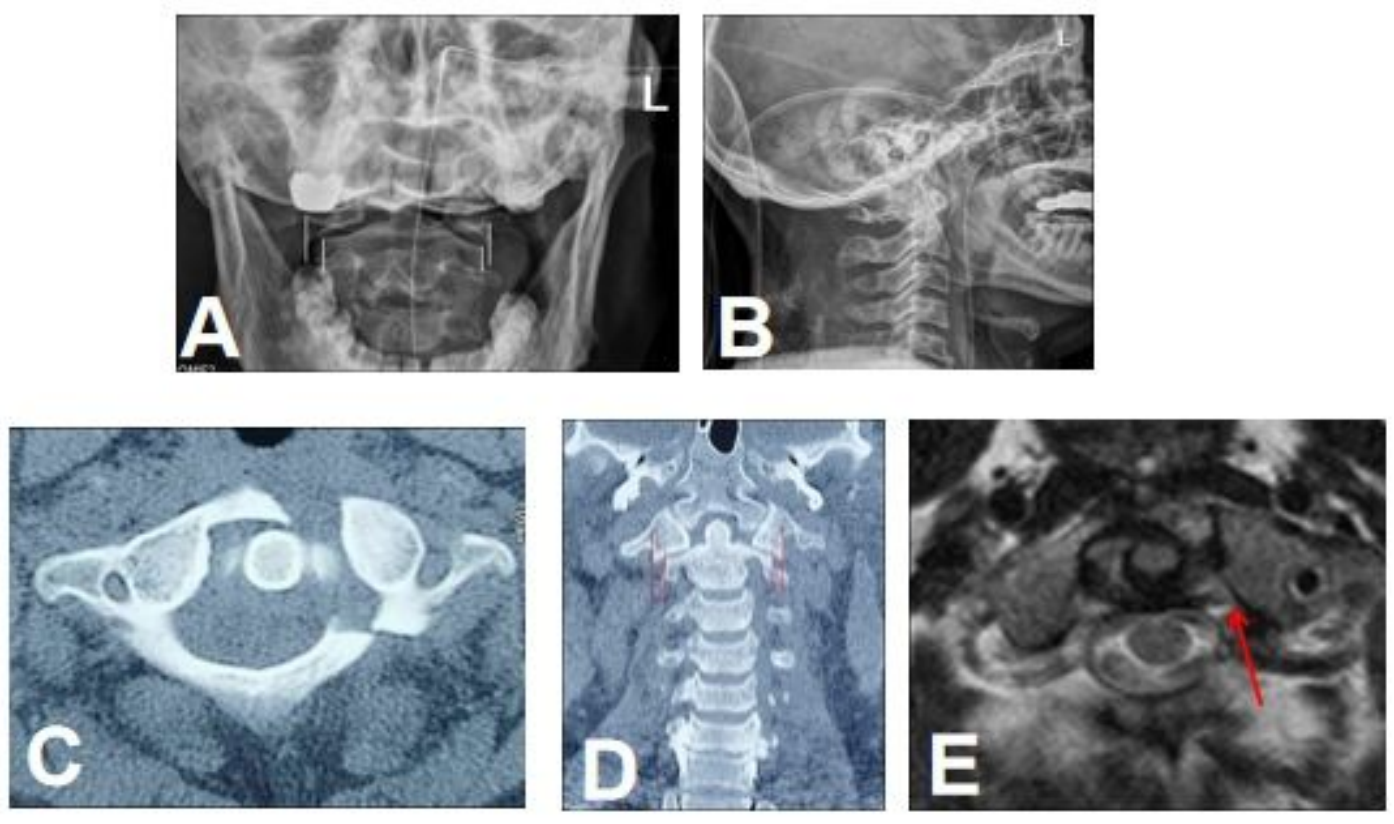

\section{Figure 1}

A 67-year-old male with $C 1$ fracture. (A, B, C, D) Preoperative X-ray and CT showed C1 fracture. (C) There were fracture line in both anterior and posterior arches (Gehweiler type III). The LMD value measured on the open X-ray film was $7.28 \mathrm{~mm}(\mathrm{~A})$, and the value measured on the coronal CT film was $6.54 \mathrm{~mm}$ (D). The MRI showed that the TAL had high signal (red arrow) at the attachment point of the left lateral mass, considering an avulsion injury (Dickman type $邓)$. While compression of spinal cord was not observed (E).
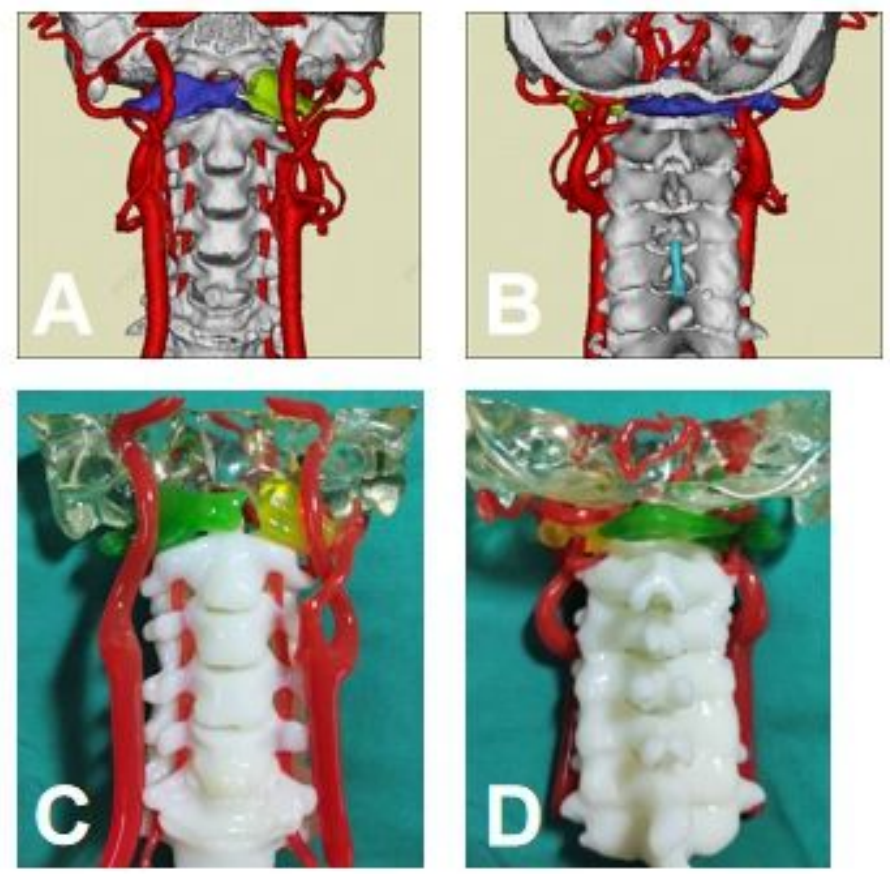

Figure 2 
3D reconstruction of anatomical digital model $(A, B)$ and 3D-printed scale 1:1 model of cervical spine (C, D). The model shows that the fracture line of $\mathrm{C} 1$ was located at the junction of the left lateral mass and the anterior and posterior arches, and the fractured end (green and yellow, respectively) is shifted outwards $(C, D)$.
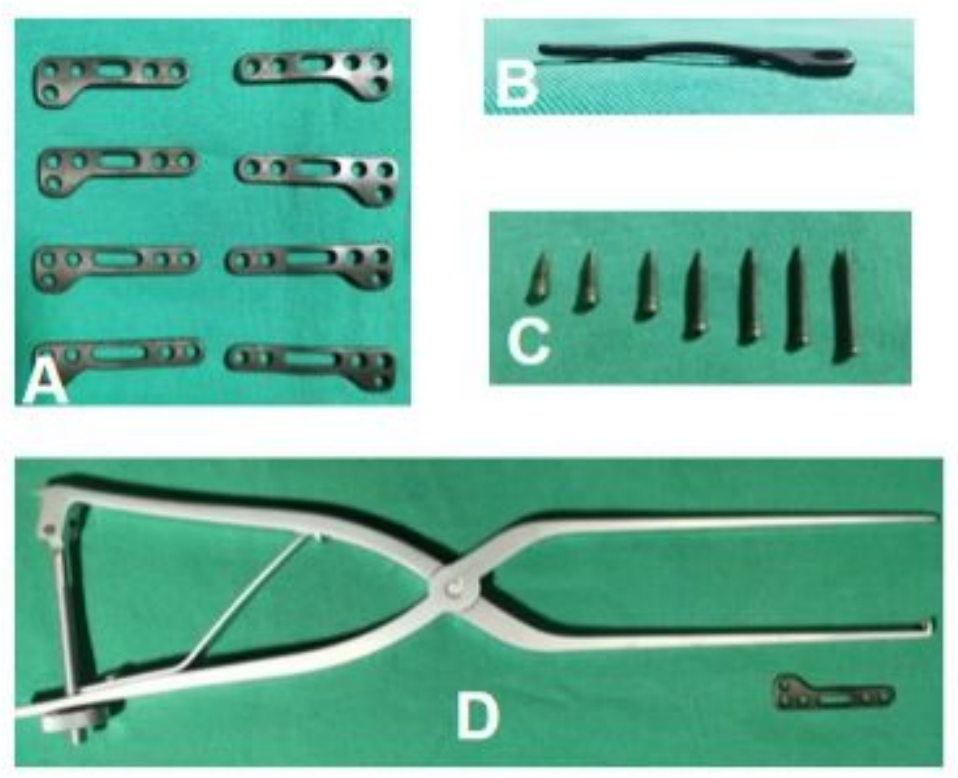

\section{Figure 3}

Different views of JeRP system. Top and side views of titanium alloy plate (A, B). There are two types of L-shaped plates, left and right, which are used for $\mathrm{C} 1$ fractures with the fracture line closed to the left or right lateral mass (A). The plate has a slight arc, fitting the physiological curvature of $C 1$ (B). Different sizes of self-tapping screws (C) and JeRP specialized repositor were shown (D).
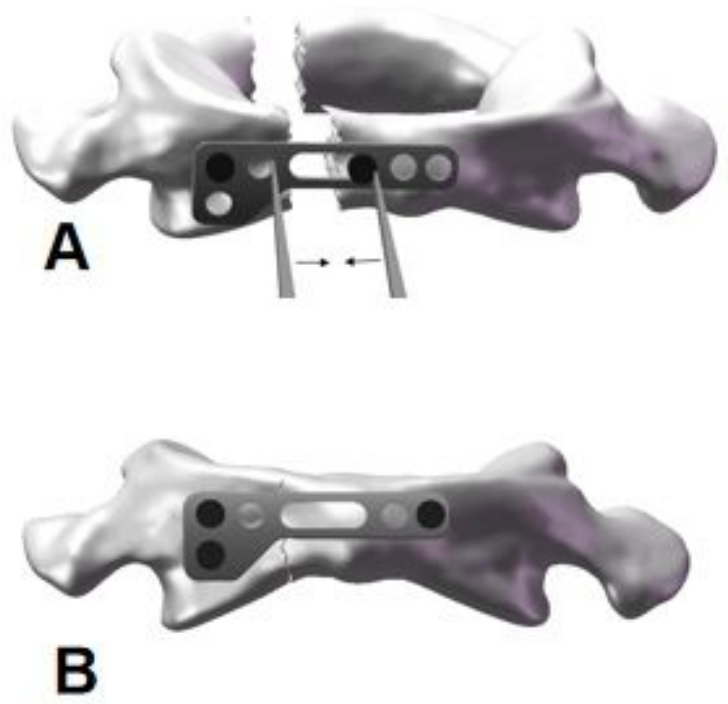

Figure 4 
Schematic diagram of JeRP plate treating C1 fracture. (A) JeRP-plate is placed in the center of the anterior arch of $\mathrm{C} 1$, and screw in the upper screw on the lateral mass close to the fracture line. $\mathrm{A}$ provisional reduction screw is screwed into the long-elliptic hole, and the nut is about $3 \mathrm{~mm}$ away from the bone surface of the anterior arch. The JeRP specialized repositor is used to compress the plate and the provisional screw. (B) After making the fracture end fit tightly, the other lateral mass screws are tightened, the temporary screw removing, and the entire reduction fixation process is complete.
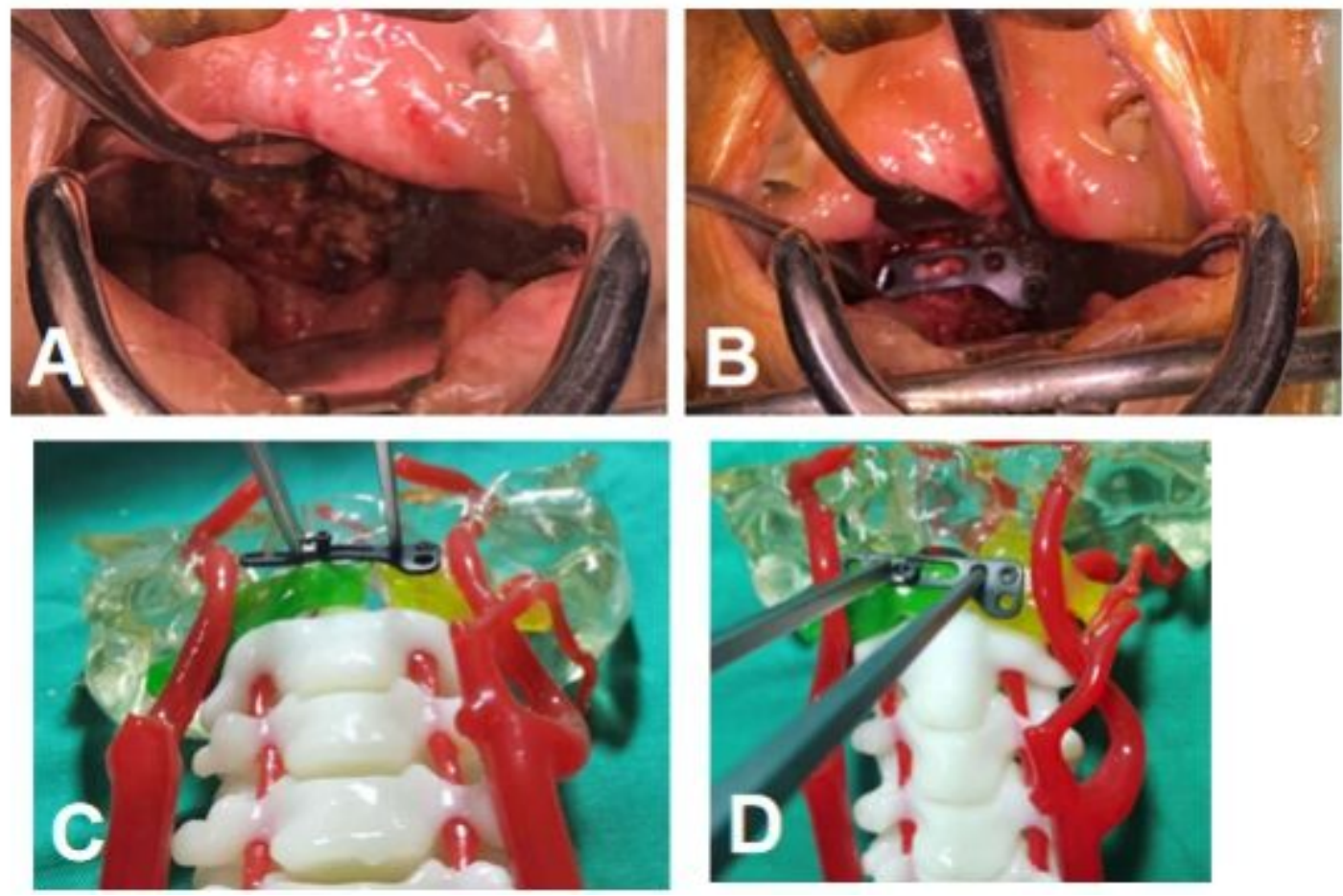

\section{Figure 5}

Surgical Technique of JeRP system. Soft tissue was dissected to fully exposed the surgical field, including anterior arch, fragment bone and lateral mass of atlas (A). The JeRP -plate was placed in anterior aspect of atlas (B). Surgery simulation on 3D printed solid model before surgery (C, D). 

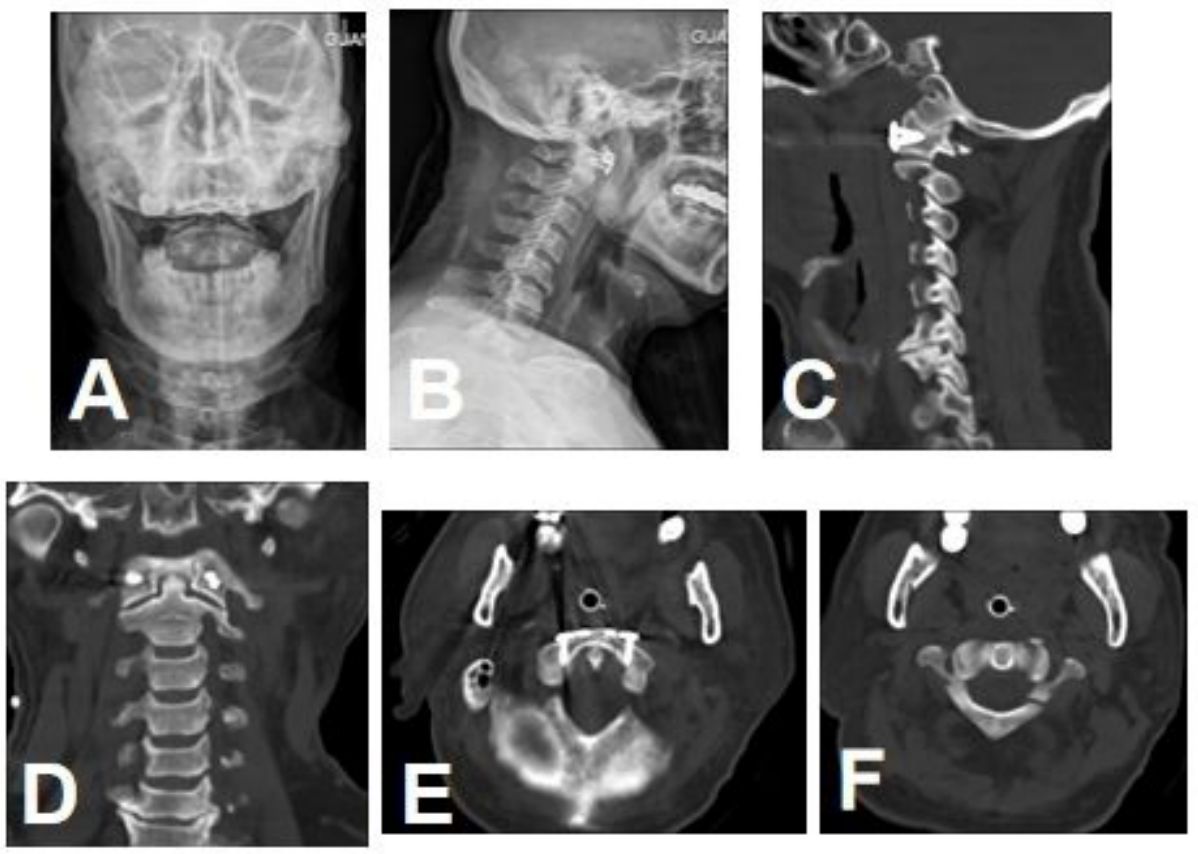

\section{Figure 6}

Postoperative X-ray and CT showed good position of the JeRP -plate (A, B, C, D,E). CT scan showed that the preoperative LMD decreased from $6.54 \mathrm{~mm}$ to $0 \mathrm{~mm}$ postoperatively (D) , and $C 1$ fracture was anatomically reduced $(E, F)$. 